EPJ manuscript No.

(will be inserted by the editor)

\title{
Phase chirality and stereo-selective swelling of cholesteric elastomers
}

\author{
S. Courty, A.R. Tajbakhsh and E.M. Terentjev \\ Cavendish Laboratory, University of Cambridge, Madingley Road, Cambridge CB3 0HE, U.K.
}

August 23, 2018

\begin{abstract}
Cholesteric elastomers possess a macroscopic "phase chirality" as the director $\boldsymbol{n}$ rotates in a helical fashion along an optical axis $z$ and can be described by a chiral order parameter $\alpha$. This parameter can be tuned by changing the helix pitch $p$ and/or the elastic properties of the network. The cholesterics also possess a local nematic order, changing with temperature or during solvent swelling. In this paper, by measuring the power of optical rotation $d \Psi / d z$, we discover how these two parameters vary as functions of temperature or solvent adsorbed by the network. The main result is a finding of pronounced stereo-selectivity of cholesteric elastomers, demonstrating itself in the retention of the "correct" chirality component of a racemic solvent. It has been possible to quantify the amount of such stereo-separation, as the basic dynamics of the effect.
\end{abstract}

PACS. 33.55.Ad Optical activity, optical rotation; circular dichroism - 61.30.Vx Polymer liquid crystals - 87.80.Pa Morphometry and stereology

\section{Introduction}

The nature appears to be inherently chiral. From the atomic scale with asymmetric carbon, to much larger length scales - like our hands or even spiral galaxies, all have the same common feature of lacking the inversion symmetry, while not characterised by any vector (dipolar) property. In other words, many natural objects are non-superimposable with their mirror image and define a pair of opposite handedness, right and left. This is the notion of chirality. Since ' its first discovery in 1848 by Pasteur [1] and attempts on mathematical abstraction by Kelvin 2, chirality and more particularly molecular chirality have always been a source of interest in various fields, from mathematics to medicine. Even though it is now much better understood, this breaking of symmetry is still an active and exciting field of research across disciplines. It is important to realise that handedness is not an absolute concept; its quantitative characteristics depend on the property being observed [3], the origin of many questions and disagreements between different groups of results.

A word on terminology is due here. There are several ways of describing chiral substances, developed in chemistry. The Rosanoff (1906) notation distinguishes between $\mathrm{D}[+]$ (for dextra) and $\mathrm{L}[-]$ (for laevo) on the basis of relative arrangement four different bonds of the chiral carbon. The Cahn-Ingold-Prelog (1956) notation is also based on ranking of bonds according to specific sequence rules, improved such that it can be used for more complex molecules; it specifies $\mathrm{R}[+]$ (for rectus, clockwise rotation) and $\mathrm{S}[-]$ (for sinister, anticlockwise). Sometimes differ- ent sources of organic chemistry data have mixed notations, e.g. the Aldrich catalogue quotes cholesterol derivatives as R-(-), with specific optical rotation $[\alpha]_{D}=-40^{\circ}$. This simply reflects the fact that different chirality indices (scalar and tensorial) may be introduced to describe different physical responses, while the proper notation is not yet developed in spite of many recent advances. So, the sense of steric chirality (asymmetry in the geometric shape of the object) is not necessarily the same as that of the third-order dielectric polarisability $\beta_{i j k}$ (determined by electronic structure) and that, in turn, may be different at different frequencies. As a result, the chiral intermolecular interaction may not be of the same handedness as, e.g., the rotation of light polarisation. The macroscopic "phase chirality" of cholesteric structure studied here is a result of cooperative action of all such effects and we simply distinguish it by macroscopic optical rotation, right-handed (clockwise, R) or left-handed (anticlockwise, L).

Chirality has a dramatic impact on most aspects of life, as enantiomers often have differences or even opposite properties (e.g. different odours, different biological functionality, toxicity, etc). This is a real problem for pharmaceutical industry, or foods and cosmetics, since the synthesis of a chemical compound leads to a racemic mixture (equal proportions of right- and left-handed enantiomers) and the stereo-selection is always a very difficult task. The reason for the difficulty lies in the fact that a pair of enantiomers differs in shape and electronic properties only in a very subtle way, which results in small corrections only to high-order molecular polarisability. As a result the molecular interactions that are sensitive to the handedness are 
Courty, Tajbakhsh and Terentjev: Stereo-selectivity of cholesteric elastomers

always very weak. New methods have been developed recently to measure such forces, for example, by detecting a difference in adhesion between an AFM tip coated with chiral molecules and the left- or right-handed substrate [5].

One of the main techniques in the field of chiral stereoseparation is column chromatography, in which a racemic mixture diffuses at slightly different rates through a silica gel coated with a molecular layer of specific chirality. Recently, a new concept of stereo-selection was introduced, based on the macroscopic phase chirality in topologically imprinted cholesteric networks [6]7. If one were to quantify the phase chirality, a corresponding order parameter has to be introduced that would reflect this symmetry. One traditional example is the cholesteric helix in liquid crystalline systems, where the molecular units are locally aligned, on average, in a uniaxial (nematic) fashion, but with the director coherently and periodically rotating over a larger length scale (helical twist), Fig. In analysing the imprinted cholesteric elastomers, a system that may retain the phase chirality while not having any on the molecular level [8], Mao and Warner (MW) have introduced a parameter that measures the (inverse) strength of imprinted helicity in the polymer network, $\alpha=\sqrt{K_{2} / D_{1}} q_{0}$, where $K_{2}$ is the Frank (twist) elastic constant [9], $q_{0}$ is the helix wavenumber at network formation and $D_{1}$ is the relativerotation coupling constant [10]. If the network is formed with a large $\alpha$, it would not be able to sustain its helical twisting when the chiral molecular moieties are removed, while at $\alpha \ll 1$ the more rigid elastic network retains most of the imprinted helix.

There are several ways of monitoring the state of phase chirality in a cholesteric material. A traditional method based on selective reflection at a certain wavelength of light is not chirality specific. In fact, recent studies has shown that one can generate the bandgap for both rightand left- circular polarisations of incoming light by only a slight mechanical deformation of cholesteric elastomers 11.12. Instead we concentrate on the effect of optical rotation. The classical optical activity (Faraday effect) of a solution of chiral molecules can be enhanced by up to $10^{4}$ times in a cholesteric phase generated by the same amount of chiral dopant $\Phi$ 9,13. As the helical pitch $p=2 \pi / q$ is inversely proportional to the concentration of chiral dopant $\Phi$ (see section 2 below), the cholesteric phase would be very sensitive to any small variation of this concentration in the network, resulting in uniform twist-untwist changes.

In this paper, we will show how to utilise this macroscopic enhancement of normally weak chiral interactions to generate stereo-selectivity of cholesteric elastomers with respect to different components of a racemic solvent. In contrast to the earlier work on imprinted networks, here we study the naturally cholesteric elastomers, where the chiral molecular moieties are a part of the network. Therefore, we cannot separate the effects of molecular chiral interactions, and those due to the phase chirality, so cleanly as in imprinted systems. However, since the results are broadly similar to those reported in [7, we believe the

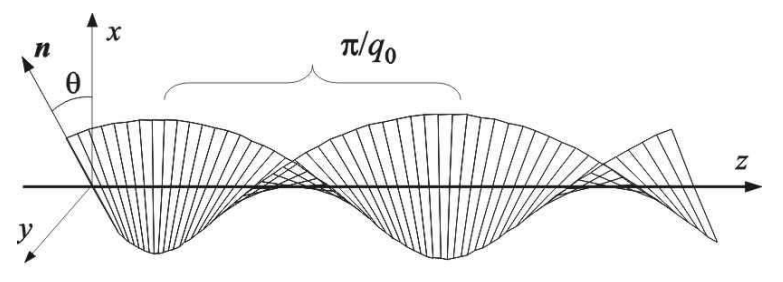

Fig. 1. Spatial distribution of the director $\boldsymbol{n}$ in an ideal cholesteric helix along the optical axis $z$. Because of the local quadrupolar symmetry of the nematic order, the periodicity interval is only the half-pitch, $\pi / q_{0}$.

macroscopic coherence of order parameter modulations in the helix plays a dominant role. In any case, for practical purposes of developing an efficient system for stereoselective separation of racemic solvents, the cholesteric elastomers could be a preferred option due to the ease of their preparation.

One central issue we shall be struggling with throughout this work is obvious, but has not been systematically examined in this context before. As one adds a solvent to a liquid crystalline network, whether a racemic mixture or a general achiral solvent, the magnitude of the local nematic order parameter changes (usually, decreases). This results in a rapid change in local optical birefringence (affecting the optical rotation) and also the strength of phase chirality (reducing the specific interaction with chiral solvent). As soon as the material becomes isotropic, i.e. loses its coherent cholesteric structure altogether, it also loses the stereo-selectivity (at least to the accuracy of our detection methods). This, in a way, is a proof that the phase chirality determines the stereo-selective swelling, and not the specific interaction with molecular moieties (which are still there in the isotropic phase). In much of the section [4 we shall be challenged by the competition between this local liquid crystalline order and the macroscopic phase chirality, aiming to develop a set of analytical tools to quantify the results.

\section{Theoretical background}

Locally cholesteric is an amorphous uniaxially ordered medium, like a nematic liquid crystal, described by the local order parameter $Q_{i j}=Q\left(n_{i} n_{j}-\frac{1}{3} \delta_{i j}\right)$. However, on larger scales the director $\boldsymbol{n}$ is a periodic modulated function of coordinates, in the ideal state rotating along a single axis $z: n_{x}=\cos \theta, n_{y}=\sin \theta, n_{z}=0$. With it rotates the local dielectric ellipsoid, with principal refractive indices $m_{\mathrm{o}}$ along the director and $m_{\mathrm{e}}$ perpendicular to $\boldsymbol{n}$ (the dielectric anisotropy $\Delta m=m_{\mathrm{o}}-m_{\mathrm{e}}$ is directly proportional to the magnitude of order parameter $Q$ ). In the classical cholesteric helix the azimuthal angle is $\theta=q_{0} z$, with the corresponding pitch $p_{0}=2 \pi / q_{0}$, see Fig. 1 The breaking of inversion symmetry results from the presence of chiral molecular groups in the material. If the elastomer network is crosslinked in this state, freezing in the helical pitch $p_{0}$, any further change in the concentration of chiral 
groups in the network would give rise to the elastic free energy of the form:

$$
F_{\mathrm{el}}=\int \frac{1}{2}\left[K_{2}\left(\frac{d}{d z} \theta-q\right)^{2}+D_{1} \sin ^{2}\left(\theta-q_{0} z\right)\right] d z,
$$

per unit area in the $x-y$ plane. Both terms represent the penalty for deviating from the initial state with $\theta=q_{0} z$. The rubber-elastic contribution simply records the conformation at network crosslinking as its ground state. The Frank term has a minimum determined by the current preferred state of phase chirality: the average helix wavenumber $q=2 \pi /\langle p\rangle=4 \pi \beta \Phi$, where $\Phi$ is the total concentration of chiral molecular groups in the material (assumed small to maintain the linear relationship $q \propto \Phi$ ) and the coefficient $\beta$ is the measure of microscopic twisting power of these groups $[9$.

Since in this paper we are mostly concerned with the effects of swelling by solvents added to the crosslinked elastomer network, two more physical effects have to be taken into account. The swollen network resists to stretching of its chains; if the overall volume is increased from $V_{0}$ to $V=V_{0}(1+\phi)$ due to an extra concentration of added solvent $\phi$, the effective dilatation strain $\gamma \approx(1-\phi)^{-1 / 3}$ contributes to the rubber elasticity. When a small concentration $\phi$ of "impurity" is added to the mesogenic system, the phase transition temperature shifts down in a linear fashion, $T^{*} \approx T_{c}(1-\kappa \phi)$. As a result, the additional free energy arises (per unit area):

$$
\begin{aligned}
F_{\mathrm{s}} & =\int \frac{1}{2}\left[3 \mu\left[(1-\phi)^{-2 / 3}-1\right]+A_{\circ}\left[T-T^{*}(\phi)\right] Q^{2}\right] d z \\
& \approx \int \frac{1}{2}\left[2 \mu \phi+A_{\circ}\left[T-T_{c}(1-\kappa \phi)\right] Q^{2}\right] d z
\end{aligned}
$$

Here $\mu$ is the rubber modulus. The ( $\phi$-dependent) $Q^{2}$ term shows the leading contribution to the thermodynamic Landau-de Gennes expansion in powers of the local order parameter. One also has to consider the mixing entropy and the coupling due to the Flory $\chi$ interaction parameters and thus complete the analysis of swelling by solvents. In fact, the problem is much more delicate. In an anisotropic material one cannot assume the simple volume change - instead the principal directions (along $z$ and in the $x-y$ plane) would stretch by slightly different factors, dependent on the nematic order through chain anisotropy [14]. Also, MW show [6] that the coupling constant $D_{1}$ is renormalised on swelling. However, the values of swelling and uniaxial strain in our experiments are so small that the corrections are only minor, reaching $2 \%$ at the most; accordingly we neglect this additional anisotropy and many complexities and subtle physical effects associated with it.

When the solvent added to the cholesteric elastomer is achiral (not optically active) the only effect on the pitch is through the affine expansion of sample dimension along $z$. In taking this view we assume that the average pitch does not change with the reduction of order parameter $Q(\phi)$. One may not regard it obvious, or even correct, because there is a large literature on cholesteric liquid crystals showing the variation of pitch with, e.g., temperature.
Our assumption is supported, at least in our elastomers, by the direct observation of nearly constant pitch, quoted in Fig. [6] below, see also the literature data [15].

When we work with a racemic mixture of right- and left-handed enantiomers and generate an imbalance between the components, $\Delta \phi=\phi_{L}-\phi_{R}$ (with the total $\phi=\phi_{L}+\phi_{R}$ ), the additional molecular chirality modifies the cholesteric pitch as well:

$$
\langle p\rangle \equiv \frac{2 \pi}{\langle q\rangle}=2 \pi\left[(1-\phi)^{1 / 3} q_{0}-q_{\mathrm{s}} \Delta \phi\right]^{-1},
$$

where $q_{\mathrm{s}}$ is a coefficient measuring the interaction of specific chirality of the solvent with the network. Note that the affine expansion term in (31) assumes that all three dimensions of the network are swelling in the same proportion, $\gamma=(1-\phi)^{-1 / 3}$. In our experiments, the samples will conserve their area in the $x-y$ plane and would only change thickness along $z$, by $\gamma_{z}=(1+\phi)$, thus modifying equation (3).

Our purpose in this paper is to explore the stereoselectivity of a cholesteric elastomer, leading to the imbalance $\Delta \phi$ of enantiomers swelling the network, by independently monitoring the weight and shape of the sample (providing the data on total $\phi$ ) and the changes in optical rotation (giving direct access to $\Delta \phi$ ). In order to interpret the results we need to revise the classical results of de Vries 13] on the rotating power of cholesteric helix.

By solving the eigenproblem for electromagnetic waves propagating along $z$ one finds the superpositions of circularly polarised plane waves of opposite signs. Their phase difference gives the optical rotation $\Psi$ in the medium, or its rotating power per unit length along $z$. The problem is, in fact, much more delicate than it is frequently presented in the literature, because of the need to correctly treat the boundary conditions for the incident linearly polarised light, see e.g. [16]. Another important issue, not treated well in the original de Vries' approach, is the limit of vanishing phase chirality, $\langle q\rangle \rightarrow 0$. This corresponds to the so-called Mauguin limit, or the 'waveguide regime', when $\langle p\rangle \Delta m \gg \Lambda_{0} / \bar{m}$, with $\Lambda_{0}$ is the wavelength of incident light and $\bar{m}=\sqrt{\bar{\varepsilon}}=\sqrt{ } \frac{1}{2}\left(\varepsilon_{\|}+\varepsilon_{\perp}\right)$ the average refractive index of the material. Not going into great detail of these complicated problems with over 30 years of history, for our practical purpose of analysing the rotation of plane polarisation in a cholesteric elastomer we shall use a simplified result for the rotation rate ('rotatory power') $d \Psi / d z$. Fortunately, in our system, the parameters combine in such a way that we never cross the bandgap. Accordingly, the difference between the full de Vries solution,

$$
\begin{aligned}
\frac{d \Psi}{d z}=\frac{2 \pi}{\langle p\rangle}(1+ & \frac{1}{2 \lambda^{\prime}}\left[\sqrt{1+\lambda^{\prime 2}-\sqrt{\delta^{2}+4 \lambda^{\prime 2}}}\right. \\
& \left.\left.-\sqrt{1+\lambda^{\prime 2}+\sqrt{\delta^{2}+4 \lambda^{\prime 2}}}\right]\right),
\end{aligned}
$$

and its more familiar approximate expansion $[9,13$ is minor. In Eq. (4) the combination $\lambda^{\prime}=\Lambda_{0} /\langle p\rangle \bar{m}$ is the nondimensional ratio of the light wavelength to the pitch, 


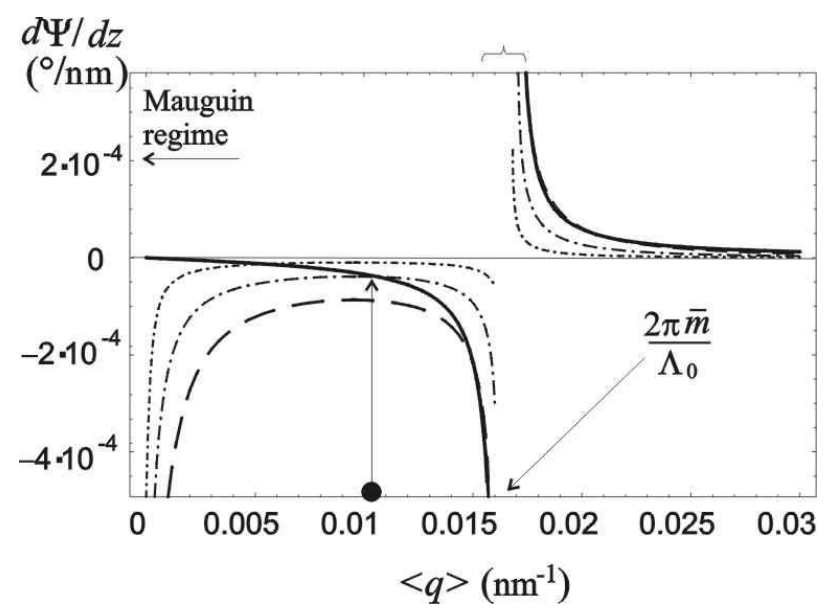

Fig. 2. The rate of optical rotation $d \Psi / d z$, as function of the helix wavenumber $\langle q\rangle=2 \pi \bar{m} \lambda^{\prime} / \Lambda_{0}$. The solid line shows the interpolated result with correct limiting behaviour. The broken lines show the classical de Vries plots for decreasing local birefringence $(\Delta m=0.15, \Delta m=0.05$ and $\Delta m=0.01)$. The bandgap is at $\lambda^{\prime}=1$, with a width decreasing with $\Delta m$. The dot marks the initial cholesteric pitch $p_{0}=580 \mathrm{~nm}$.

and $\delta=\left(\varepsilon_{\perp}-\varepsilon_{\|}\right) /\left(\varepsilon_{\perp}+\varepsilon_{\|}\right) \approx \Delta m / \bar{m}$ is the parameter of relative dielectric anisotropy. In our material the extraordinary and ordinary refractive indices are, respectively, $m_{\mathrm{e}}=1.75$ and $m_{\mathrm{o}}=1.6$ (making $\bar{m}=1.68$ and the light wavelength in the medium $\left.\Lambda_{0} / \bar{m}=377 \mathrm{~nm}\right)$. For $\lambda^{\prime}=\sqrt{1 \pm \delta}$ a dispersion anomaly appears in (4) in the form of Bragg-like reflection; this full solution does not have a divergence for $d \Psi / d z$ but a finite value on the edges of a bandgap of width $\delta$ [13. In our case this anomaly would center at $\langle p\rangle \approx 377 \mathrm{~nm}$. The analysis below will show that the initial pitch of the cholesteric helix is $p_{0} \approx 580 \mathrm{~nm}$, which means that our range of measurements is always on the longer wavelength side of the bandgap.

Figure 2 shows the variation of the rotatory power with the helix wavenumber (or equivalently, with $\lambda^{\prime}$ for fixed $\Lambda_{0}$ ) for the parameters of our experimental system. We plot the function that interpolates between the correct behaviour near the bandgap and the required linear decrease at $q \rightarrow 0$. For comparison, a sequence of de Vries curves is also plotted, for a series of decreasing $\Delta m$ (and the underlying nematic order parameter $Q$, directly proportional to it).

Equation (4) and all its modifications contain two important parameters which are changing in our experiments: the relative dielectric anisotropy $\Delta m$ and the effective pitch length $\langle p\rangle$. The first of these changes with temperature and swelling with small-molecule 'impurities' and reflects the local (nematic) liquid crystalline ordering. The changing effective pitch, or the helical wavenumber $\langle q\rangle=$ $2 \pi /\langle p\rangle$, reflects the phase chirality of a cholesteric and is determined by chiral imbalance between the components of racemic mixture swelling the cholesteric network. Rotating power is an unambiguous measure of phase chirality (as opposed to, say, selective reflection spectrum which can be too broad in cholesteric networks and also present in both right- and left-handed modes for the same material [12]). We shall use Eq. (4) to extract the effective pitch from the measurements of optical rotation. For this, let us re-write it explicitly showing the two relevant parameters, $\Delta m$ and $\langle p\rangle$, for $\delta \ll 1$ :

$$
\frac{d \Psi}{d z} \approx-\frac{\pi \bar{m}^{2} \Delta m^{2}\langle p\rangle^{3}}{2 \Lambda_{0}^{2}\left(\bar{m}^{2}\langle p\rangle^{2}-\Lambda_{0}^{2}\right)} .
$$

To find $\langle p\rangle$ we need to resolve this cubic equation, which gives the approximate result in the relevant region of parameters, represented by the solid line in Fig. 2]

$$
\langle p\rangle \approx-\frac{\pi \Delta m^{2}+\sqrt{\pi^{2} \Delta m^{4}+16 \bar{m}^{2} \Lambda_{0}^{2}(d \Psi / d z)^{2}}}{4 \bar{m}^{2}(d \Psi / d z)}
$$

This interpolated model will serve us for the rest of this work, to help extracting the values of effective cholesteric pitch, as a measure of phase chirality, from the measured $d \Psi / d z$ and the deduced $\Delta m$.

\section{Methods}

\subsection{Sample preparation}

The cholesteric liquid crystal elastomer was synthesised following the general method introduced by Kim and Finkelmann [17. Siloxane backbone chains were reacted under centrifugation at 7000rpm with $90 \mathrm{~mol} \%$ mesogenic side groups (the mixture of nematic 4-pentylphenyl-4'-(4buteneoxy)benzoate, labelled PBB, and cholesterol pentenoate, ChP, in proportion $4: 1)$ and $10 \mathrm{~mol} \%$ of $1,4 \mathrm{di}(11-$ undeceneoxy)benzene, di-11UB, crosslinker groups for 45 minutes at $75^{\circ} \mathrm{C}$ to form a partially crosslinked gel. For the further 4 hours the reaction proceeded under centrifugation at $60^{\circ} \mathrm{C}$, during which time the solvent was allowed to evaporate, leading to an anisotropic deswelling of the gel and completion of crosslinking. All of the volume change in this setup occurs by reducing the thickness of the gel, while keeping the lateral dimensions fixed (due to centrifugation): this introduces a very strong effective biaxial extension in the $x-y$ plane. At this second-stage temperature of $60^{\circ} \mathrm{C}$ the dried polymer is in the cholesteric phase and its director is forced to remain in the plane of stretching - this results in the uniform cholesteric texture which is finally crosslinked at this second stage of preparation. The chemical composition of our networks is shown in Fig. 3.

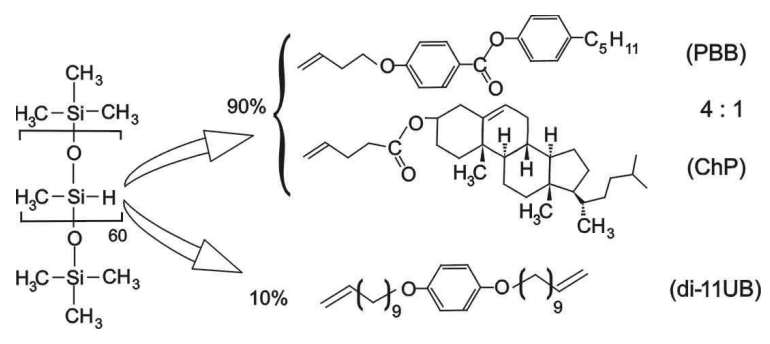

Fig. 3. Chemical composition of the cholesteric network. 


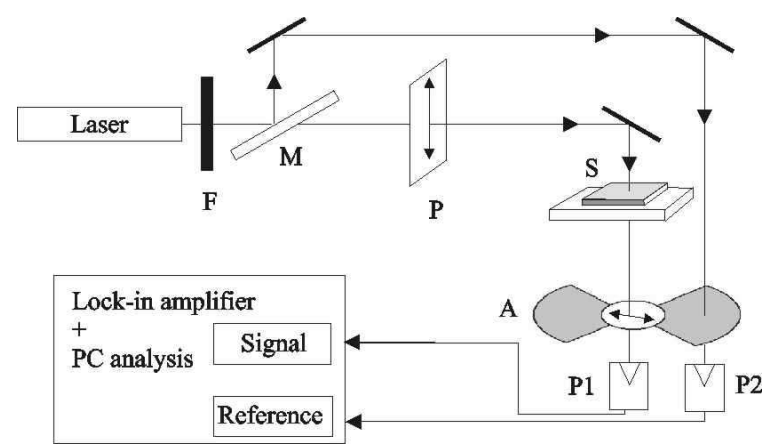

Fig. 4. Experimental set-up for measuring the optical rotation $\Psi$. (F): neutral density filter; (M): beam splitter, (P): GlanThomson polariser; $(\mathrm{S})$ : glass cover slip with sample; (A): rotating analyzer with light chopper; $(\mathrm{P} 1, \mathrm{P} 2)$ : photodiodes linked to the lock-in amplifier.

Differential scanning calorimetry measurements (PerkinElmer Pyris 7 DSC) were used to characterise the resulting elastomer. The glass transition was unambiguously determined at $T_{g} \approx-10^{\circ} \mathrm{C}$ and the clearing point, the isotropic-cholesteric transition occurs at $T_{\mathrm{c}} \approx 90^{\circ} \mathrm{C}$. No additional thermal transitions were found between these two critical temperatures. All experiments were performed at room temperature, sufficiently far from both transitions (except when we studied the variation of optical rotation with order parameter, for comparison).

\subsection{Experimental set-up}

The optical rotation $\Psi$ can be determined experimentally by using a dynamical method 18 based on measuring the phase difference between the split parts of linearly polarised laser beam (He-Ne laser, $\lambda_{\text {laser }}=633 \mathrm{~nm}, 30 \mathrm{~mW}$, from Melles-Griot), one passing through the sample and the rotating analyzer (fixed frequency $\sim 16 \mathrm{~Hz}$ ), the other through the optical chopper (providing the reference signal to lock on), Fig. 团 The phase difference $\Delta \Theta$ between the two beams is measured by an integer number of periods with a lock-in amplifier (Stanford Research) and corresponds directly to the optical rotation $\Psi$ from which the effective cholesteric pitch $\langle p\rangle$ is then calculated. The elastomer is deposited onto a glass coverslip (area conservation will be observed) and a solvent droplet of known volume $(10 \mu l)$ is placed on it, with the beam spot in the middle. The solvents used for achiral and racemic environment are respectively toluene:hexane mixture (ratio 1:6, from Acros) and 2-Bromopentane (from Acros).

The optical configuration described above has been also used to measure the birefringence $\Delta m$ of a very similar nematic elastomer during solvent evaporation. From this we obtained an independent data on $\Delta m$ to use in the analysis based on Eq. (6), assuming that the local properties of cholesteric are approximately the same as of the corresponding nematic. In the measurements of optical rotation we do not care about the ellipticity of light out of the sample, only about the angle of the principal axis. In the $\Delta m$ measurements of a uniformly birefringent medium we do. The optimal configuration is to send linearly polarised light at an angle of $\pi / 4$ to the director and then place a $\lambda / 4$ plate after the sample with one of its axis adjusted to be parallel to the incident polarisation (to recover the linear polarisation) [19]. The relative phase difference $\Delta \Theta$ between the perpendicular and parallel polarisations is directly related to $\Delta m$ by $\Delta \Theta=2 \pi d \Delta m / \Lambda_{0}$, with $d$ the independently measured sample thickness.

For temperature measurements, the glass coverslip is directly placed onto a hot stage connected to a controller (Stanton Redcroft) with a range of temperature varying from $20^{\circ} \mathrm{C}$ to $200^{\circ} \mathrm{C}$.

\section{Results and discussion}

\subsection{Optical rotation}

Figure[5] shows the evolution with time of the optical rotation from cholesteric elastomer after solvent swelling and subsequent drying. Initially the value of $\Psi$ is constant and is determined by the initial pitch $p_{0}$ of the dry cholesteric state and the local nematic order defined by $\Delta m_{0}$. At $t=0$ a drop of solvent is placed in the beam path, as labelled by arrow in the plot. The optical rotation rapidly drops to zero because the amount of solvent taken into the network exceeds the concentration $\phi^{*}$ at which the material becomes isotropic, $Q(T, \phi)=0$. Accordingly, the rotatory power vanishes - see Eq. (5) with $\Delta m=0$. The spectacular difference between the two types of solvent is observed on subsequent slow evaporation. As the concentration of the achiral solvent in the swollen network decreases, the liquid crystalline order returns back (cf. Fig. 7 below for detail) and the observed optical rotation gradually approaches the same level it had in the initial dry cholesteric elastomer. As the total concentration $\phi$ of the racemic solvent decreases, the rotatory power returns,

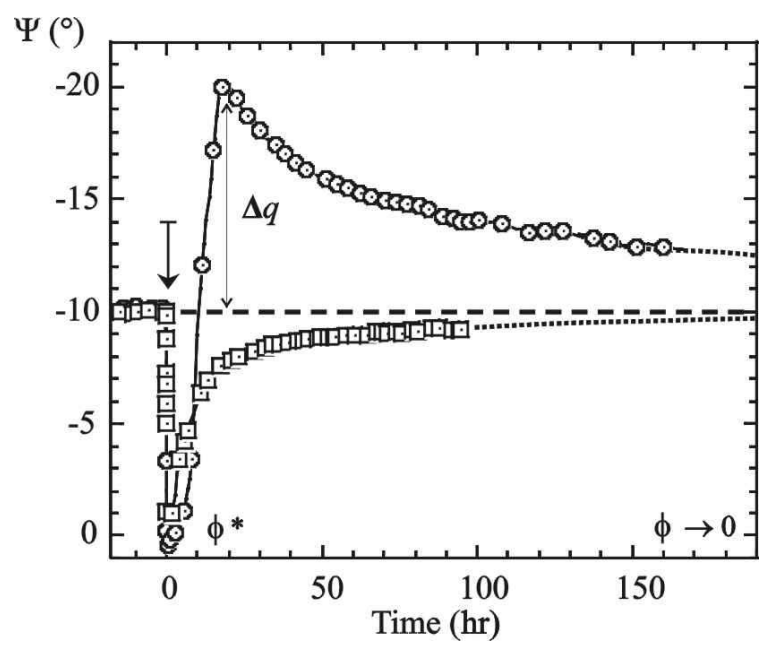

Fig. 5. Variation of total angle $\Psi$ of rotation of incident linear polarisation after swelling of cholesteric elastomer with achiral (squares) and racemic solvent (circles). The arrow represents the time deposition of the solvent droplet onto the sample. 
but its magnitude is significantly higher than in the dry cholesteric. After a maximum at $\sim 20 \mathrm{hr}$, even after a very long time, the network does not return to its original helical state, but saturates asymptotically at $\Psi \approx-12^{\circ}$. This rise of optical rotation above its initial value, and the whole non-monotonic time (and indirectly - concentration) dependence, are the signature of chiral separation of solvent components. The network retains the component that matches its own phase chirality, while letting the other component evaporate - as a result the effective helical power increases. This is labelled by $\Delta q$ in the plot, to make connection with Eq. (3) and Fig. 9] below.

The behavior presented on figure. 5 is remarkable and represents the central result of this work. Our challenge is now to quantitatively analyse the effect of stereo-selectivity of cholesteric elastomers. We need to extract from the optical data, $d \Psi / d z$, the information on how the pitch $\langle p\rangle$ varies as function of the solvent $\phi$ retained by the network. For this, we need to know the relationship between the effective pitch $\langle p\rangle$ and the local order $Q$ (or $\Delta m$ ) and as the network approaches the transition into an isotropic state, first by heating above $T_{c}$ and secondly, by swelling above $\phi^{*}$. This will lead to a way to determine the current cholesteric pitch any small variation of solvent in the network and to relate it to chiral imbalance $\Delta \phi$ of guest molecules in the network. Finally, we can monitor the overall amount of solvent in the network by simply measuring the weight of samples as function of time. This will provide the independent data on $\phi(t)=\phi_{L}+\phi_{R}$. In addition, this is important to provide the information about the current thickness of the swollen network, $d(t)$, which is necessary to convert the raw rotation angle $\Psi$ into the rotatory power $d \Psi / d z$; since our samples have their $x-y$ area conserved, the thickness change is simply $d(t)=d_{0}(1+\phi)$.

\subsection{Local order parameter}

It is unfortunately nearly impossible to independently measure the local birefringence $\Delta m$, or equivalently, the local nematic order parameter $Q$, of a cholesteric liquid crystal. The difficulty is the same as to measure $Q$ in a polydomain texture. Perhaps the only experimental technique that could offer access to such local information is NMR, relating the angularly averaged line splitting to local bias of probe molecule orientation, affected by the nematic mean field. In our case, all we can do is to measure $\Delta m$ on a chemically similar nematic liquid crystal system and assume that its value and variation would be the same in a cholesteric. It is not a totally unreasonable assumption: the degree of nematic order is very reliably $Q \sim 0.5 \pm 0.1$ for most nematic liquid crystal materials (apart from main-chain polymers, which is not our case). The refractive indices depend more strongly on the molecular structure, varying between, say, 1.45 and 1.85 in different nematic materials.

We chose a composition of nematic elastomer as close to that in Fig. 3 as we could: with PBB and the same amount of di-11UB crosslinker, with hopefully a similar

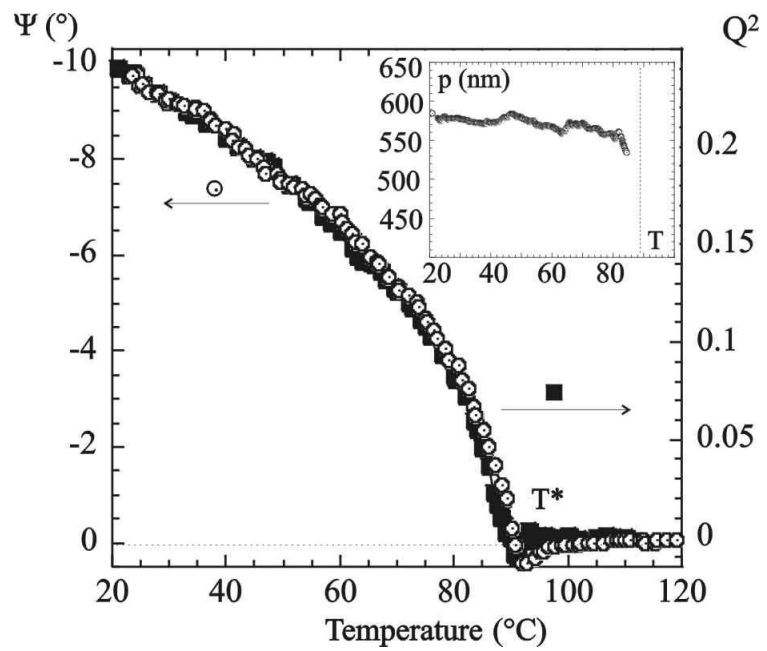

Fig. 6. Superposition of $\Psi$ (circles) and $Q^{2}$ (dark squares) as function of the temperature, from room temperature to $T^{*}$. In inset, dependence of the pitch $p$ with the temperature, proving that the nematic order parameter has no strong effect on the average cholesteric pitch, at least in our elastomers.

molecular anisotropy and the amount of polarisable $\pi$ electrons. As a confirmation of our choice, the clearing temperature of this material, $T_{c} \approx 90^{\circ} \mathrm{C}$, was similar to that of the cholesteric. Making an aligned monodomain nematic elastomer with this chemical composition, we then measure its birefringence as function of temperature. Since the anisotropy of dielectric tensor is directly related to the nematic order parameter, $\Delta m=$ const $\cdot Q[9]$, we obtain the latter by calibrating the proportionality constant at room temperature against a separate X-ray measurement of $Q$.

Figure 6 gives the results and the corresponding analysis. We plot, against temperature, the square of the nematic order parameter, $Q^{2}$, determined as described above (filled squares, right $y$-axis). This shows the expected behaviour, reported and discussed many times in the recent literature 10. The data for $Q(T)$ can be very well fitted by an empirical critical dependence $Q=0.8\left(1-T / T_{c}\right)^{0.28}$ (an interesting and provocative observation of its own, also discussed in the literature). This data set is compared with the result plotted on the left $y$-axis (open circles), for the optical rotation of our cholesteric elastomer as it was heated towards its isotropic phase (note, the initial rotation of $\sim 10^{\circ}$ is the same as that in Fig. 5). The matching is quite spectacular, including even the pretransitional region where both $\Psi$ and $\Delta m$ effectively change sign, proving that $\Psi \propto Q^{2}$, which is the result predicted by Eq. (5).

This exact matching of optical rotation $\Psi(T)$ and the local birefringence $\Delta m(T)$ also proves that the other parameters in the Eq. (5), in particular the cholesteric pitch $\langle p\rangle$, do not significantly change with the nematic order. The inset in Fig. 6] shows the effective pitch calculated from the data for $\Psi$ and $\Delta m$ by our method outlined in section 2 It clearly does not vary very much, in spite of the underlying nematic order continuously dropping to 


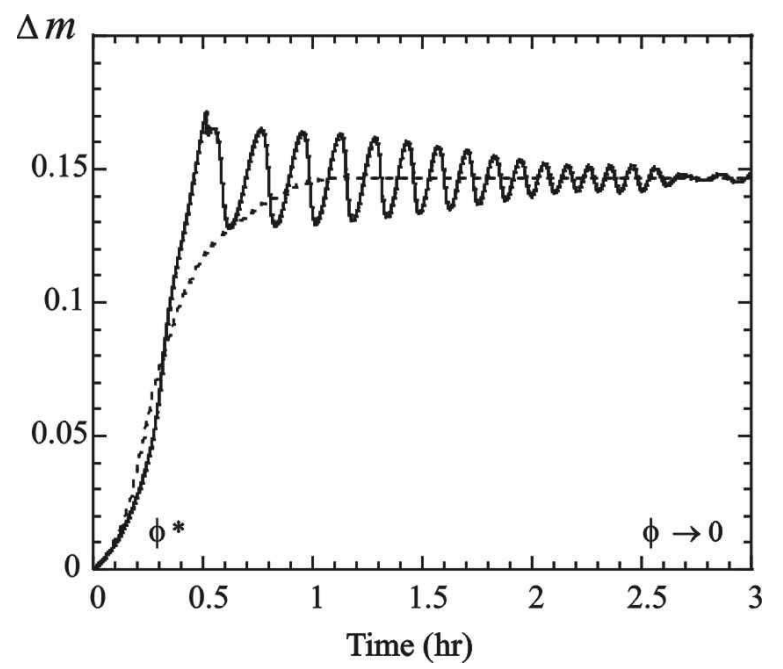

Fig. 7. Variation of the birefringence $\Delta m$ on solvent evaporation from the nematic elastomer, between the isotropic phase $\left(\right.$ at $\left.\phi^{*}, t=0\right)$ to the nematic phase $(\phi \rightarrow 0)$. The dotted line represents a smoothed model curve for $\Delta m(t)$.

zero. Such a conclusion is not unexpected in cholesteric elastomers where the helix is crosslinked into the rubbery network, and has been reported before [15].

Now, what will happen if the cholesteric network is swollen with a non-chiral small molecule solvent, at constant temperature? First of all, as for the temperature, we need to know what is the dependence of the underlying local order parameter $Q$ on the solvent concentration $\phi$. It was assumed that, for low $\phi$, the effect of added impurities is to linearly shift down the transition temperature $T_{c}=$ $T^{*}(\phi)$, see Eq. (2). It is easy to find the critical solvent concentration at which the material becomes isotropic, $\phi^{*} \approx 8 \%$ in our case, giving the parameter $\kappa \approx 2.3$. Accordingly, we might have expected that the order parameter follows the law $Q(\phi)=0.8\left(1-T / T_{c}[1-\kappa \phi]\right)^{0.28}$, with the variation exactly as that shown in Fig. 6 Instead, we directly measured $\Delta m$ as function of time as the droplet of solvent deposited on the nematic sample (see the configuration Fig. 4) swells the network and then gradually evaporates. Figure 7 presents the result, in terms of $\Delta m$ rather than $Q$, with the time $t=0$ chosen at the moment when the sample first returns to its birefringent state (at $\phi^{*} \approx 8 \%$, measured independently). As the solvent evaporates further $(\phi \rightarrow 0)$, the birefringence increases and eventually returns to its initial value demonstrating the complete restoration of the nematic order.

The actual experimental result shown in Fig. [7 is very interesting and rather unexpected, and will certainly lead to a separate investigation. We observe periodic oscillation of $\Delta m$ as $\phi \rightarrow 0$ in a nematic elastomer deswelling, while constrained on a substrate. We hypothesise that these oscillations are caused by the concentration gradient as one side of the sample is attached to a glass substrate (and its area conserved); as the solvent evaporates from the free surface, it also causes a gradient of $Q$, and with it - the mechanical strains in the network, which attempts to uniaxially expand along $\boldsymbol{n}$ with the strain magnitude $\propto Q$.
Clearly, the set of coupled differential equations for the time evolution of local $\phi, Q$ and elastic strain has an oscillating instability. Note that the cholesteric network, in our main experiments on swelling and optical rotation is also attached to a glass substrate, so presumably a similar effect should occur there.

Although tempted, here we do not discuss this phenomenon further, not to distract the reader from the main theme of this paper. For our analysis of effective helical pitch/wavenumber as a measure of phase chirality, we need to substitute the raw data for $d \Psi / d z$ obtained from Fig. 5 and the data for $\Delta m$ from Fig. 7 into the Eq. (6). If one compares the time scales, it becomes clear that the curious oscillating regime of $\Delta m(t)$ occurs at a very early stage of deswelling, in a region where $\Psi \approx 0$ in Fig. 5 Accordingly, for the main (and most interesting) bulk of data we could use a simpler, smoothed model for $\Delta m(t)$ which is shown as a dotted line in Fig. [7] We obtained such a model in a somewhat arbitrary fashion, trying to match as closely as possible the data and the linear model for $Q(T, \phi)$; however, the main point of this argument is that in the region of main interest the deviation of the model from the raw data is not relevant.

\subsection{Quantifying the stereo-selectivity}

We obtain the same results for changes in local order parameter swelling by a racemic solvent and calculate, from the Eq. (6), how the effective helical pitch $\langle\tilde{p}\rangle$ varies with time. The last task remaining, to successfully map thus obtained $\langle p(t)\rangle$ on the important concentration dependence, is to independently measure the change in total concentration $\phi=\phi(t)$ as the network gradually loses the solvent. Figure 8 shows the result of such weight measurement of our cholesteric sample initially swollen in a large amount of each corresponding solvent. Such an experiment is, necessarily, much less accurate than the rest of our data nevertheless the results appear unambiguously pointing at a different saturation level at $t \rightarrow \infty$.

In fact, the detailed analysis of the decay of total concentration of solvent in a swollen cholesteric network shows another basic and interesting result, the discussion of which is beyond the scope of this paper. We attempted to fit the data for $\phi(t)$ (below $\phi^{*}$, in the mesophase) and the only good fit was achieved by a "stretched exponential" function, the same for both solvents (with a relaxation time slightly different, which is only natural for chemically different solvents):

$$
\begin{aligned}
\text { achiral : } \phi(t) & =C e^{-\left(t / \tau_{\mathrm{a}}\right)^{2 / 3}} \\
\text { racemic : } \phi(t) & =C e^{-\left(t / \tau_{\mathrm{r}}\right)^{2 / 3}}+\Delta \phi,
\end{aligned}
$$

with $\tau_{\mathrm{a}} \approx 2.6$ and $\tau_{\mathrm{r}} \approx 1.5 \mathrm{~h}$, but with characteristically the same prefactor $C \approx 14$ (in \% units, as in Fig. 8). Such a time dependence of average concentration during surface evaporation is very different from classical predictions [20], offering the law $\phi=\left(8 \phi_{0} / \pi^{2}\right) \exp \left[-\pi^{2} D t / 8 d^{2}\right]$. 


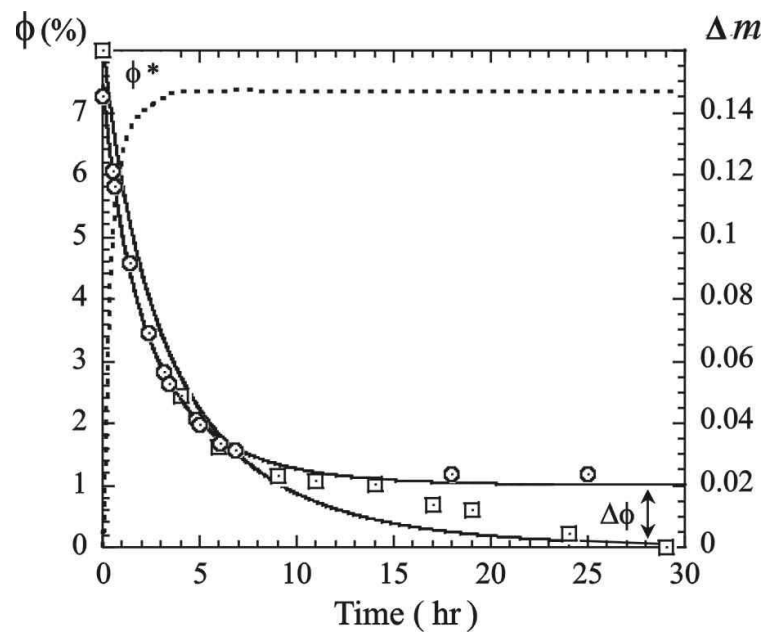

Fig. 8. Time evolution of total concentration $\phi(t)$ as the solvent evaporates from the cholesteric network. As in Fig. [5] squares represent the achiral solvent and circles - the racemic mixture. The time $t=0$ is chosen, as in Fig. [7 as a moment when the material first re-enters the liquid crystalline state (at $\phi^{*} \approx 8 \%$. Solid lines are the fit by a stretched-exponential model (see text). The right $y$-axis shows the parallel data for birefringence $\Delta m(t)$ (dotted line, representing the smoothed model in Fig. (7).

Such a deviation could be due to coupled nonlinear effects of elastic strain, order $Q$ and inhomogeneous solvent concentration across the sample layer.

Having mapped the data for total concentration $\phi(t)$ [and the associated sample thickness decrease, $d_{0}(1+\phi)$ ] on the results for optical rotation, $\Psi(t)$ and local birefringence $\Delta m(t)$, we can obtain the concentration dependence of phase chirality in our system. Figure 9 presents the results of our analysis, based on the Eq. (6), for the variation of $\langle\tilde{p}\rangle=2 \pi /\langle q\rangle$ as function of concentration. ${ }^{1}$ The data

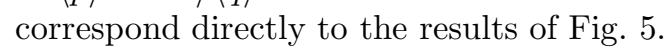

It appears that, unlike for the temperature effect illustrated in Fig. [6] a continuous unwinding of the natural helix occurs on swelling in achiral solvent. This is not an affine effect of increasing sample thickness: the $d(t)$ variation has been accounted for in evaluating the rotation rate $d \Psi / d z$. The likely reason for such an effect is the dilution of molecular chirality and its reducing effect on the macroscopic scale.

The main effect we are interested in is the stereoselective response to the racemic solvent. The marked increase in the effective phase chirality and the failure of the solvent to evaporate completely (also demonstrated in Fig. 8) are clear indications of the network selecting and retaining the solvent component with the chirality sense matching that of the helix. We could reasonably assume that all the retained solvent is left-handed (L), so the $\Delta \phi(t \rightarrow \infty)=\phi_{L} \approx 1 \%$. Note, however, that the strength of chiral solvent retention only become noticeable

\footnotetext{
1 The results in the region of phase transition, close to $\phi^{*}$, are too ambiguous because of several independent critical functions (especially $\Delta m$ ) and not shown in the plot
}

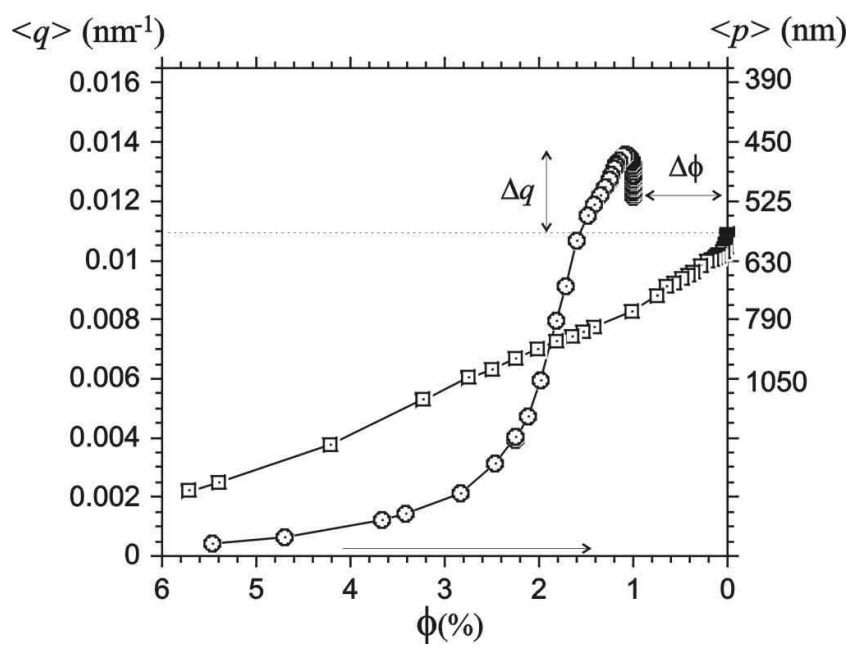

Fig. 9. Variation of phase chirality, represented by the helix wavenumber $\langle q\rangle$ (or, equivalently, the pitch $\langle\tilde{p}\rangle$ on the right $y$ axis) as function of decreasing concentration $\phi$, in achiral (data labelled by squares) and racemic (circles) environments. The increase in $\Delta q$ and the retention of chiral solvent component $\Delta \phi$ are indicated on the plot.

when the overall solvent content $\phi$ reduces below $\sim 3 \%$. At this level, the local order parameter $Q$ (expressed as $\Delta m$ in Figs. (7) and [8) increases to its nearly saturation value characteristic of the dry network. When the local nematic order is weak, at higher $\phi$, we see no stereo-selectivity. This indicates the role of phase chirality in the observed phenomenon, as well as the method of extracting the Lsolvent trapped in the network: one simply needs to heat the material to its isotropic state, or mechanically stretch above the critical strain so that the helix is unwound [10.

Comparing the final values of $\Delta q$ and $\Delta \phi$, we can deduce another phenomenological parameter used in the MW theory, the coefficient $q_{\mathrm{s}}$ in the Eq. (3). After the affine expansion effect is eliminated, as in Fig. 9 the remaining linear relation gives $q_{\mathrm{s}} \approx 0.1 \mathrm{~nm}^{-1}$.

\section{Conclusions}

In summary, we found a strong and reproducible effect of stereo-selectivity in cholesteric elastomers. The study has unambiguously pointed to the macroscopic phase chirality (the cholesteric helix) as the dominant force behind the phenomenon. From a combination of analytical tools provided by the theory of optical rotation in cholesterics and the local analogy between the cholesteric and the nematic elastomer systems, we were able to quantitatively follow the variation of helical pitch with solvent concentration. Comparing the effects of an ordinary achiral solvent and the racemic mixture of two opposite chiral small-molecule components, we demonstrate how the cholesteric elastomer selectively retains the component with the matching sense of chirality.

Most of our experimental work was based on delicate optical measurements and required monodomain cholesteric 
Courty, Tajbakhsh and Terentjev: Stereo-selectivity of cholesteric elastomers

(and nematic) elastomers. Preparation and the resulting quality of these could present many practical difficulties. However, for the purpose of stereo-selectivity, one does not need monodomain networks! Once we have demonstrated and studied the effect in model samples, one can now proceed to develop a new technology of chiral separation using cholesteric polymer networks of different chemical composition (to control overall solubility in target racemic solvents and to choose the desired handedness of the helix). These networks do not have to be aligned in any way; perhaps the best practical way is to prepare highly porous sponges with high internal surface area, which could then be used to extract chiral components from racemic mixtures (and release them on, e.g., subsequent heating). However, our results indicate that careful monitoring of timing and overall solvent content has to be maintained in order for such a "chiral sponge" to remain in its most effective regime (at high local $Q$ ). Numerous applications of this effect in biomedical industry and stereo-selective sensing come to mind.

Several fundamental and interesting problems were left behind. The most startling is the oscillating effect of solvent loss under mechanical constraints, but even the basic effects of diffusion in the medium with coupled non-linear degrees of freedom are challenging for both theory and experiment.

We acknowledge many useful discussions with M. Warner and P. Cicuta, and the financial support from EPSRC.

\section{References}

1. L. Pasteur, Ann. Chim. Phys. 24, 442 (1848).

2. W. Thomson, in Baltimore Lectures (C. J. Clay \& Sons, London, 1904).

3. M. Osipov, B. Pickup, and D. Dunmur, Mol. Phys. 84, 1193 (1995).

4. A. Harris, R. Kamien, and T. Lubensky, Rev. Mod. Phys. 71, 1745 (1999).

5. R. McKendry, M. Theoclitou, T. Rayment, and C. Abell, Nature 391, 566 (1998).

6. Y. Mao and M. Warner, Phys. Rev. Lett. 86, 5309 (2001).

7. S. Courty, A. Tajbakhsh, and E. Terentjev, Phys. Rev. Lett. LD9109, (2003).

8. Y. Mao and M. Warner, Phys. Rev. Lett. 84, 5335 (2000).

9. P.-G. de Gennes and J. Prost, The Physics of Liquid Crystals (Oxford University Press, Oxford, 1994).

10. M. Warner and E. Terentjev, Liquid Crystal Elastomers (Oxford University Press, Oxford, 2003).

11. P. Bermel and M. Warner, Phys. Rev. E 65, 056614 (2002).

12. P. Cicuta, A. Tajbakhsh, and E. Terentjev, Phys. Rev. E 65, 051704 (2002).

13. H. de Vries, Acta Cryst. 4, 219 (1951).

14. X.-J. Wang and M. Warner, Macromol. Theor. Simul. 6, 37 (1997).

15. G. Maxein, S. Mayer, and R. Zentel, Macromolecules 32, 5747 (1999).

16. V. Belyakov, V. Dmitrienko, and V. Orlov, Sov. Phys. Uspekhi 22, 63 (1979).
17. S. Kim and H. Finkelmann, Macromol. Rapid Commun. 22, 429 (2001).

18. C. Hunte, P. Gibbs, and U. Singh, Am. J. Phys. 69, 91 (2001).

19. K. Lim and J. Ho, Mol. Cryst. Liq. Cryst. 47, 173 (1978).

20. J. Crank, The Mathematics of Diffusion (Oxford University Press, Oxford, 1975). 